\title{
CHARGE DENSITIES OF THE NEUTRON AND PROTON
}

\author{
Gerald A. Miller \\ Department of Physics, \\ University of Washington \\ Seattle, Washington 98195-1560
}

\begin{abstract}
A model-independent analysis of the infinite-momentum-frame charge density of partons in the transverse plane is presented for the nucleon. We find that the neutron parton charge density is negative at the center, so that the square of the transverse charge radius is positive, in contrast with many expectations. Additionally, the proton's central $u$ quark charge density is larger than that of the $d$ quark by about $70 \%$. The proton (neutron) charge density has a long range positively (negatively) charged component.
\end{abstract}

Keywords: Generalized Parton Distributions; Form Factors, Quark Densities 
A truly impressive level of experimental technique, effort and ingenuity has been brought to measuring the electromagnetic form factors of the proton and neutron (nucleon) [1]. These quantities are probability amplitudes that the nucleon can absorb a given amount of momentum and remain in the ground state, and as such should be important sources of information about the nucleon charge and magnetization densities.

The text-book interpretation of these form factors is that their Fourier transforms are measurements of the charge and magnetization densities. But the initial and final nucleons have different momentum, and therefore different wave functions. This is because the relativistic boost operator that transforms a nucleon at rest into a moving one changes the wave function in a manner that depends on the momentum of the nucleon. The presence of different wave functions of the initial and final nucleons invalidates a probability or density interpretation.

A proper determination of a charge density requires that the quantity be related to the square of a wave function or of a field operator. The technical solution to the problem of determining the relevant density operator has been known for a long time [2], and has been elegantly explained recently [3, 4] in terms of generalized parton distributions GPDs. The charge density $\rho(\mathbf{b})$ [5] of partons in the transverse plane is a two-dimensional Fourier transform of the $F_{1}$ form factor. Here we present the first phenomenological analysis of existing data to determine $\rho(\mathbf{b})$ for the neutron and proton. The results for the neutron contradict the longstanding notion, derived from both gluon-exchange and meson-cloud models [6, 7], that the non-vanishing charge density at the center of the neutron is positive.

We begin by by presenting definitions of the form factors. Let $J^{\mu}(x)$ be the electromagnetic current operator, in units of the proton charge. Then the nucleon form factors are given by

$$
\left\langle p^{\prime}, \lambda^{\prime}\left|J^{\mu}(0)\right| p, \lambda\right\rangle=\bar{u}\left(p^{\prime}, \lambda^{\prime}\right)\left(\gamma^{\mu} F_{1}\left(Q^{2}\right)+i \frac{\sigma^{\mu \alpha}}{2 M} q_{\alpha} F_{2}\left(Q^{2}\right)\right) u(p, \lambda),
$$

where the momentum transfer $q_{\alpha}=p_{\alpha}^{\prime}-p_{\alpha}$ is taken as space-like, so that $Q^{2} \equiv-q^{2}>0$. The nucleon polarization states are chosen to be those of definite light-cone helicities $\lambda, \lambda^{\prime}[8]$. The charge (Dirac) form factor is $F_{1}$, normalized such that $F_{1}(0)$ is the nucleon charge, and the magnetic (Pauli) form factor is $F_{2}$, normalized such that $F_{2}(0)$ is the anomalous magnetic moment. the Sachs form factors 9 ]

$$
G_{E}\left(Q^{2}\right) \equiv F_{1}\left(Q^{2}\right)-\frac{Q^{2}}{4 M^{2}} F_{2}\left(Q^{2}\right), G_{M}\left(Q^{2}\right) \equiv F_{1}\left(Q^{2}\right)+F_{2}\left(Q^{2}\right)
$$

were introduced so as to provide an expression for the electron-nucleon cross section (in the one photon exchange approximation) that depends on the quantities $G_{E}^{2}$ and $G_{M}^{2}$ but not the product $G_{E} G_{M}$. In the Breit frame, in which $\mathbf{p}=-\mathbf{p}^{\prime}, G_{E}$ is the nucleon helicity flip matrix element of $J^{0}$. Furthermore, the scattering of neutrons from the electron cloud of atoms measures the derivative $-d G_{E}\left(Q^{2}\right) / d Q^{2}$ at $Q^{2}=0$, widely interpreted as six times the meansquare charge radius of the neutron. However, any probability or density interpretation of $G_{E}$ is spoiled by a non-zero value of $Q^{2}$, no matter how small [10]. Any attempt to analytically incorporate relativistic corrections in a $p^{2} / m_{q}^{2}$ type of expansion would be doomed, by the presence of the quark mass, $m_{q}$, to be model-dependent.

The newly appreciated and widely studied generalized parton distributions GPDs [11] are of high current interest because they can be related to the total angular momentum carried 
by quarks in the nucleon and can be determined using deeply virtual Compton scattering experiments [12]. These distributions are specific matrix elements of quark field operators, between nucleon states, which in contrast to the usual quark distribution functions, do not have the same momenta. We consider the specific case in which the longitudinal momentum transfer $\xi$ is zero, and $\lambda^{\prime}=\lambda$. Then, in the light-cone gauge, $A^{+}=0$, the matrix element defining the GPD, $H_{q}$ for a quark of flavor $q[13]$ is

$$
H_{q}(x, t)=\int \frac{d x^{-}}{4 \pi}\left\langle p^{+}, \mathbf{p}^{\prime}, \lambda\left|\bar{q}\left(-\frac{x^{-}}{2}, \mathbf{0}\right) \gamma^{+} q\left(\frac{x^{-}}{2}, \mathbf{0}\right)\right| p^{+}, \mathbf{p}, \lambda\right\rangle e^{i x p^{+} x^{-}} .
$$

We use the abbreviation $H_{q}(x, \xi=0, t) \equiv H_{q}(x, t)$ and $-t=-\left(p^{\prime}-p\right)^{2}=\left(\mathbf{p}^{\prime}-\mathbf{p}\right)^{2}=$ $-q^{2}=Q^{2}$. The simple form of $t$ results from its invariance under transverse boosts [8]: Lorentz transformations, defined by a transverse vector $\mathbf{v}$ that transform a four-vector $k$ according to $k^{+} \rightarrow k^{+}, \mathbf{k} \rightarrow \mathbf{k}-k^{+} \mathbf{v}$ and $k^{-}$such that $k^{2}$ is unchanged. These quantities are part of a kinematic subgroup of the Poincaré group that obey the same commutation relations as those among the generators of the Galilean transformations for non-relativistic quantum mechanics in the transverse plane. The presence of the operator $\gamma^{+}$insures that independent field operators appear in the matrix element.

GPDs allow for a unified description of a number of hadronic properties [12]. The most relevant for us are that if $t=0$ they reduce to conventional PDFs $H_{q}(x, 0)=q(x)$, and that the integration of $H_{q}$ over $x$ yields the nucleon electromagnetic form factor:

$$
F_{1}(t)=\sum_{q} e_{q} \int d x H_{q}(x, t)
$$

The spatial structure of a nucleon can be examined if one uses [2, 3, 4] nucleonic states that are transversely localized. The state with transverse center of mass $\mathbf{R}$ set to 0 is formed by taking a linear superposition of states of transverse momentum. In particular,

$$
\left|p^{+}, \mathbf{R}=\mathbf{0}, \lambda\right\rangle \equiv \mathcal{N} \int \frac{d^{2} \mathbf{p}}{(2 \pi)^{2}}\left|p^{+}, \mathbf{p}, \lambda\right\rangle .
$$

where $\left|p^{+}, \mathbf{p}, \lambda\right\rangle$ are light-cone helicity eigenstates [8] and $\mathcal{N}$ is a normalization factor satisfying $|\mathcal{N}|^{2} \int \frac{d^{2} \mathbf{p}_{1}}{(2 \pi)^{2}}=1$. References [14, 15] use wave packet treatments that avoid states normalized to $\delta$ functions, but this leads to the same results as using Eq. (5). Note however, the relevant range of integration in Eq. (5) must be restricted to $|\mathbf{p}| \ll p^{+}$to maintain the interpretation of a nucleon moving with well-defined longitudinal momentum[14]. Thus we use a frame with very large $p^{+}$. It is in just such a frame that the interpretation of a nucleon as a set of a large number of partons is valid.

Using Eq. (5) sets the transverse center of momentum of a state of total very large momentum $p^{+}$to zero, so that transverse distance $\mathbf{b}$ relative to $\mathbf{R}$. can be defined. Thus we may generalize the quark-field operator appearing in Eq. (3) by making a translation:

$$
\hat{O}_{q}(x, \mathbf{b}) \equiv \int \frac{d x^{-}}{4 \pi} q_{+}^{\dagger}\left(-\frac{x^{-}}{2}, \mathbf{b}\right) q_{+}\left(\frac{x^{-}}{2}, \mathbf{b}\right) e^{i x p^{+} x^{-}} .
$$


The impact parameter dependent PDF is defined [14] as the matrix element of this operator in the state of Eq. (15):

$$
q(x, \mathbf{b}) \equiv\left\langle p^{+}, \mathbf{R}=\mathbf{0}, \lambda\left|\hat{O}_{q}(x, \mathbf{b})\right| p^{+}, \mathbf{R}=\mathbf{0}, \lambda\right\rangle .
$$

The use of Eq. (5) in Eq. (7) allows one to show that $q(x, \mathbf{b})$ is the two-dimensional Fourier transform of the GPD $H_{q}$ :

$$
q(x, \mathbf{b})=\int \frac{d^{2} q}{(2 \pi)^{2}} e^{i \mathbf{q} \cdot \mathbf{b}} H_{q}\left(x, t=-\mathbf{q}^{2}\right),
$$

with $H_{q}$ appearing because the initial and final helicities are each $\lambda$. A complete determination of $H_{q}(x, t)$ (with $t \leq 0$ ) would determine $q(x, \mathbf{b})$.

One finds a probability interpretation [2] by integrating $q(x, \mathbf{b})$ over all values of $x$. This sets the value of $x^{-}$to 0 , so that

$$
\int d x q(x, \mathbf{b}) \equiv\left\langle p^{+}, \mathbf{R}=\mathbf{0}, \lambda\left|q_{+}^{\dagger}(0, \mathbf{b}) q_{+}(0, \mathbf{b})\right| p^{+}, \mathbf{R}=\mathbf{0}, \lambda\right\rangle .
$$

If one multiplies the above relation by the quark charge $e_{q}$ (in units of $e$ ), sums over quark flavors, uses Eq. (5) with $\hat{O}_{q}(x, \mathbf{b})=e^{-i \hat{\mathbf{p}} \cdot \mathbf{b}} \hat{O}_{q}(x, \mathbf{0}) e^{i \hat{\mathbf{p}} \cdot \mathbf{b}}$ along with Eq. (4), the resulting infinitemomentum-frame IMF parton charge density in transverse space is

$$
\rho(b) \equiv \sum_{q} e_{q} \int d x q(x, \mathbf{b})=\int \frac{d^{2} q}{(2 \pi)^{2}} F_{1}\left(Q^{2}=\mathbf{q}^{2}\right) e^{i \mathbf{q} \cdot \mathbf{b}} .
$$

This transverse charge density $\rho(b)$, based on $F_{1}$ [2], seems to contrast with the lore relating the charge density to $G_{E}$. There is no conflict, a feature understood by considering the relation between the Breit frame and the IMF. In the Breit frame, the helicity flip matrix element of $J^{0}$ is $G_{E}$. One uses a Lorentz transformation to consider the form factor in the IMF. Then the operator helicity-flip matrix element of $J^{0}$ becomes the helicity non-flip matrix element of $J^{+}$, or $F_{1}$. The transformation to the IMF gains model independence while replacing $G_{E}$ by $F_{1}$.

We exploit Eq. (10) by using measured form factors to determine $\rho(b)$. Recent parameterizations [16, 17, 18] of $G_{E}$ and $G_{M}$ are very useful so we use Eq. (2) to obtain $F_{1}$ in terms of $G_{E}, G_{M}$. Then $\rho(b)$ can be expressed as a simple integral of known functions:

$$
\rho(b)=\int_{0}^{\infty} \frac{d Q Q}{2 \pi} J_{0}(Q b) \frac{G_{E}\left(Q^{2}\right)+\tau G_{M}\left(Q^{2}\right)}{1+\tau}
$$

with $\tau=\frac{Q^{2}}{4 M^{2}}$ and $J_{0}$ a cylindrical Bessel function.

A straightforward application of Eq. (11) to the proton using the parameterizations [16, 17] yields the results shown in the upper panel of Fig. 1. The curves obtained using the two different parameterizations overlap. Furthermore, there is negligible sensitivity to form factors at very high values of $Q^{2}$ that are currently unmeasured. The density is peaked at low values of $b$, but has a long positive tail, suggestive of a long-ranged, positively charged pion cloud.

The neutron results are shown in the lower panel of Fig. 1. The curves obtained using the two different parameterizations seem to overlap, but see below. The surprising result is that 

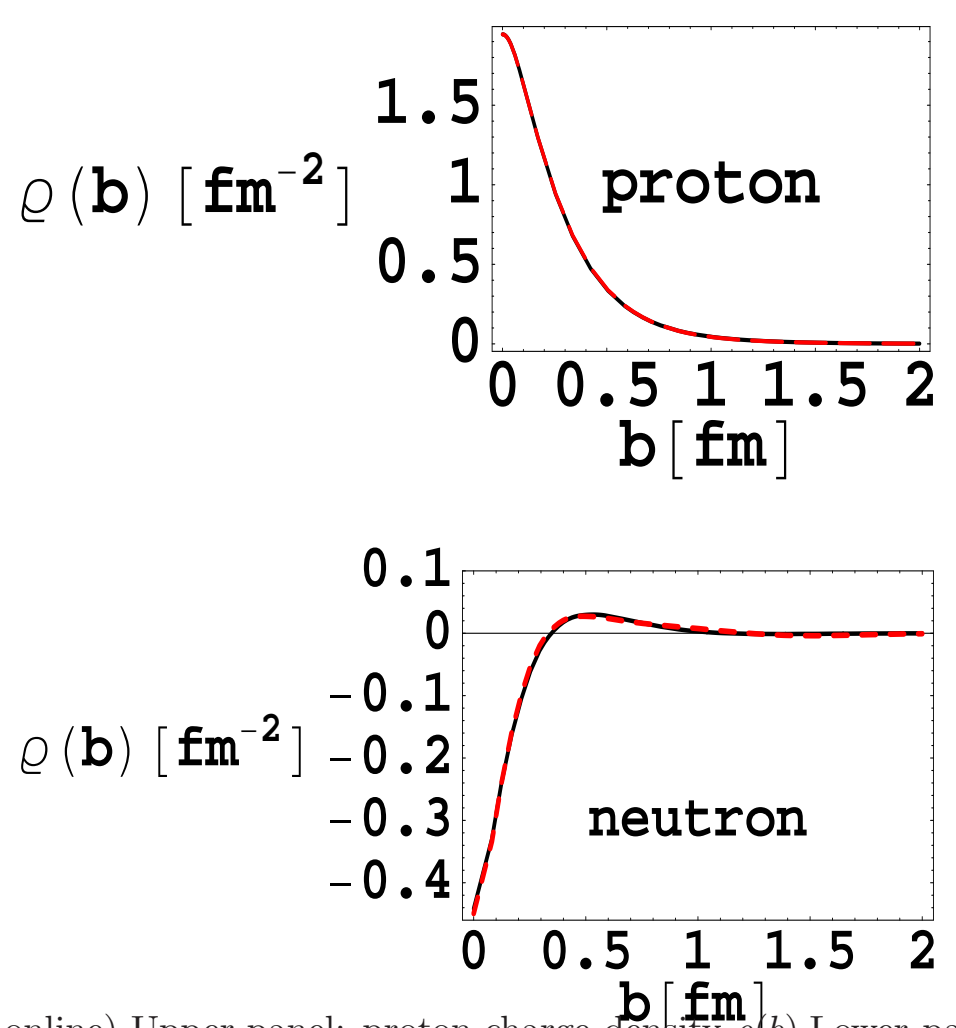

FIG. 1: (Color online) Upper panel: proton charge density $\rho(b)$.Lower panel: neutron charge density. The solid curves use the parameterization of [17], and the dashed (red) curve uses [16].

the central neutron charge density is negative. If the neutron is sometimes a proton surrounded by a negatively charged pionic cloud, one would expect to obtain a positive central density [7]. Another mechanism involving correlations in the nucleonic wave function induced by one gluon exchange would also lead to a positive central density because the interaction between two identical $d$ quarks [6] is repulsive. The values of the integral of Eq. (11) are somewhat sensitive to the regime $2<\tau<4$ for which $G_{E}$ is as yet unmeasured. About $30 \%$ of the value of $\rho(0)$ arises from this region.

The negative central density deserves further explanation. The upper panel of Fig. 2 shows $F_{1}$ for the neutron obtained using the two different parameterizations which are observably different. However, in both cases $F_{1}$ is negative (because of the dominance of the $G_{M}$ term of Eq. (11)) for all values of $Q^{2}$. This along with taking $b=0, J_{0}(Q b)=1$ in Eq. (11) leads immediately to the central negative result. The long range structure of the charge density is captured by displaying the quantity $b \rho(b)$ in the lower panel of Fig. 2. At very large distances from the center, again suggesting the existence of the long-ranged pion cloud.

The present analysis provides detailed information about the location of charge density within the nucleon, and also incorporates the lore regarding mean-square-radii, MSR. It has long been known that the MSR defined by the form factor $G_{E}$ is dominated by the Foldy term $-1.91 /\left(4 M^{2}\right)=-0.126 \mathrm{fm}^{2}[19,20]$. arising from the neutron magnetic moment $F_{2}(0)$. The experimental value of the $G_{E} \mathrm{MSR}$, cited in [17], is $(-0.114 \pm 0.003) \mathrm{fm}^{2}$, so the MSR associated with $F_{1}$ (obtained from the integral $\left.\int d^{2} b b^{2} \rho(b)\right)$ is small and positive $\left(+0.012 \mathrm{fm}^{2}\right)$. This result is consistent with Figs. 1 and 2. However, knowing the MRS of $F_{1}$ does not, by itself, allow one to conclude that the central neutron charge density is negative, does not reveal the critical model-independent feature that at the very largest distances the charge density is negative and does not imply the oscillatory behavior displayed in Figs. 1 and 2.

One can gain information about the individual $u$ and $d$ quark densities by invoking charge 

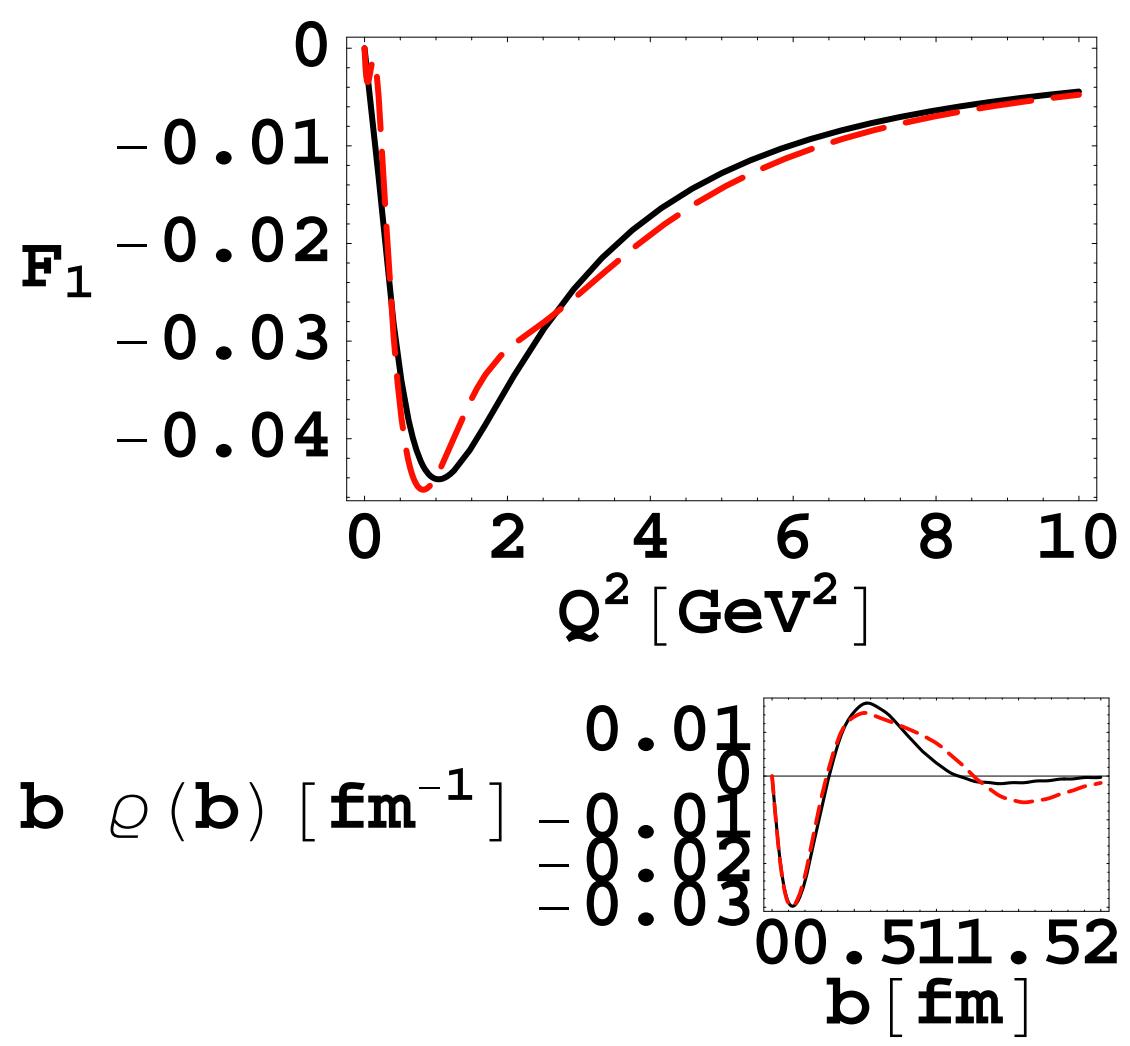

FIG. 2: Upper panel: $F_{1}$. Lower panel: $b \rho(b)$ in transverse position space. The solid curves are obtained using [17] and the dashed curves with [16].

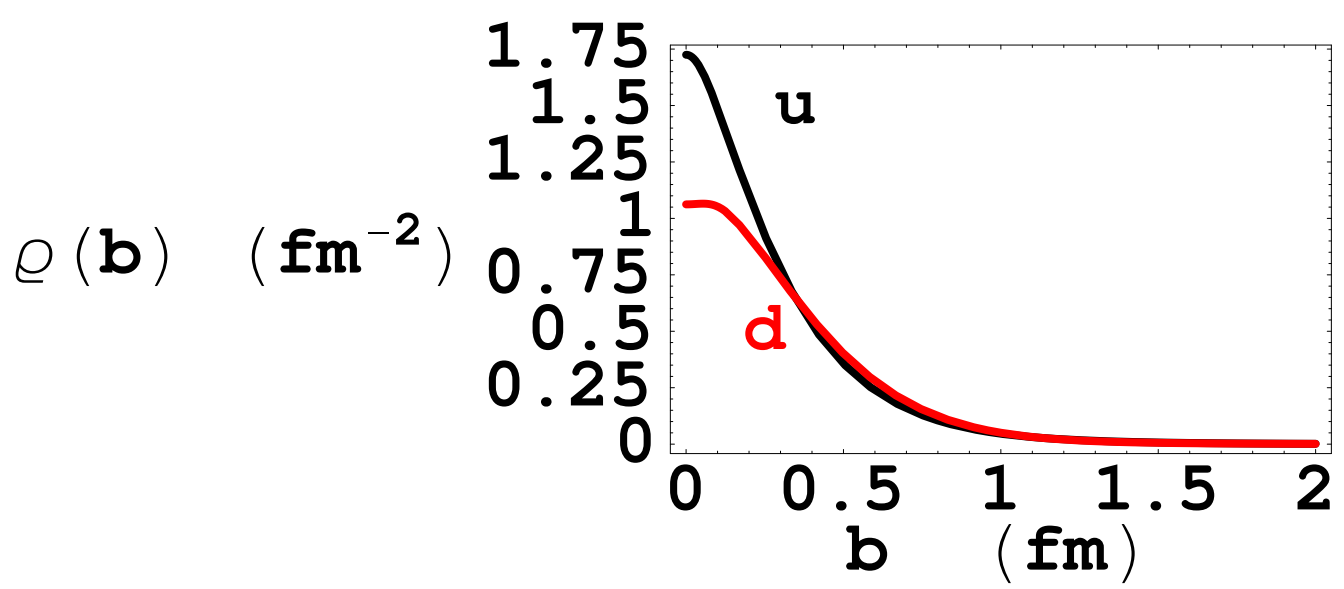

FIG. 3: Transverse densities for up $u$ and down $d$ quarks. Each is normalized to unity

symmetry (invariance under a rotation by $\pi$ about the $z$ (charge) axis in isospin space) [21] so that the $u, d$ densities in the proton are the same as the $d, u$ densities in the neutron. We also neglect the effects of $s \bar{s}$ [22] or heavier pairs of quarks. In this case $\rho_{u}(b)=\rho_{p}(b)+$ $\rho_{n}(b) / 2, \rho_{d}(b)=\rho_{p}(b)+2 \rho_{n}(b)$. The results, shown in Fig. 3, and obtained with either form factor parameterization are that the central up quark density is significantly larger than the central down quark density. The latter becomes larger at distances away from the center.

Model independent information about parton distributions has been obtained. In particular, 
the central density of the neutron is negative. Future experimental measurements of neutron electromagnetic form factors could render the present results more precise, or potentially modify them considerably. Obtaining a quantitative and intuitive understanding of our results presents a challenge to lattice QCD and to builders of phenomenological models.

I thank the USDOE (FG02-97ER41014) for partial support of this work, and J. Arrington, A. Bernstein, A. Bulgac, M. Burkardt, W. Detmold, S. D. Ellis, B. Holstein, and C. Hyde-Wright for useful discussions.

[1] H. y. Gao, Int. J. Mod. Phys. E 12, 1 (2003) [Erratum-ibid. E 12, 567 (2003)]; C. E. HydeWright and K. de Jager, Ann. Rev. Nucl. Part. Sci. 54, 217 (2004); C. F. Perdrisat, V. Punjabi and M. Vanderhaeghen, arXiv:hep-ph/0612014.

[2] D.E. Soper, Phys. Rev. D 15, 1141 (1977).

[3] M. Burkardt, Int. J. Mod. Phys. A 18, 173 (2003).

[4] M. Diehl, Eur. Phys. J. C 25, 223 (2002) [Erratum-ibid. C 31, 277 (2003)].

[5] Our notation is that $x^{ \pm} \equiv\left(x^{0} \pm x^{3}\right) / \sqrt{2}, p^{ \pm} \equiv\left(p^{0} \pm p^{3}\right) / \sqrt{2}$, and $p_{\mu} x^{\mu}=p^{-} x^{+}+p^{+} x^{-}-\mathbf{p} \cdot \mathbf{b}$. The coordinates perpendicular to the 0 and 3 directions are denoted as $\mathbf{b}$ and $\mathbf{p}$.

[6] J. L. Friar, Part. Nucl. 4, 153 (1972); R. D. Carlitz, S. D. Ellis and R. Savit, Phys. Lett. B 68, 443 (1977); N. Isgur, G. Karl and D. W. L. Sprung, Phys. Rev. D 23, 163 (1981).

[7] A. W. Thomas, S. Théberge and G. A. Miller, Phys. Rev. D 24, 216 (1981).

[8] J. Kogut and D.E. Soper, Phys. Rev. D 1, 2901 (1970).

[9] R. G. Sachs, Phys. Rev. 126, 2256 (1962)

[10] The momentum difference between the initial and final states appears via the use of derivatives of momentum-conserving delta functions in the moments computed by Ref. [9]

[11] D. Müller et al., Fortschr. Phys. 42, 101 (1994); X.Ji, J. Phys. G 24, 1181 (1998); A.V. Radyushkin, Phys. Rev. D 56, 5524 (1997); K. Goeke, M.V. Polyakov, and M. Vanderhaeghen, Prog. Part. Nucl. Phys. 47, 401 (2001).

[12] X. Ji, Phys. Rev. Lett. 78, 610 (1997).

[13] The vector current $\bar{q} \gamma^{\mu} q$ is conserved, so $F_{1}$ is independent of the renormalization scale $\mu^{2}$ [4].

[14] M. Burkardt, Phys. Rev. D 62, 071503 (R) (2000).

[15] M. Diehl et al., Nucl. Phys. B 596, 33 (2001).

[16] R. Bradford, A. Bodek, H. Budd and J. Arrington, Nucl. Phys. Proc. Suppl. 159, 127 (2006).

[17] J. J. Kelly, Phys. Rev. C 70, 068202 (2004).

[18] J. Arrington, Phys. Rev. C 69, 022201 (R) (2004).

[19] L. L. Foldy, Phys. Rev. 83, 688 (1951).

[20] U. G. Meissner, AIP Conf. Proc. 904, 142 (2007).

[21] G. A. Miller, A. K. Opper and E. J. Stephenson, Ann. Rev. Nucl. Part. Sci. 56, 253 (2006) G. A. Miller, Phys. Rev. C 57, 1492 (1998); G. A. Miller, A. W. Thomas and A. G. Williams, Phys. Rev. Lett. 56, 2567 (1986).

[22] A. Acha et al., Phys. Rev. Lett. 98, 032301 (2007). 